\title{
Relaciones entre la aplicación de los enunciados jurídicos y la motivación de las decisiones judiciales RAFAEl HERNÁNDEZ MARÍN
}

Universidad de Murcia

\begin{abstract}
RESUMEN. En el estudio de la actividad judicial, el tema fundamental no es la motivación de las decisiones judiciales, como sostiene la filosofía jurídica actual, sino la aplicación de los enunciados jurídicos. Y creo además que la concepción estándar de la motivación de las decisiones judiciales es errónea precisamente por no haber tenido en cuenta este hecho. Por otra parte, también considero equivocadas las concepciones existentes respecto a la aplicación de los enunciados jurídicos (sea la teoría del silogismo judicial, sea la concepción lógica). Según las tesis expuestas en mi libro Las obligaciones básicas de los jueces (2005), respecto a la aplicación de los enunciados jurídicos primarios, la concepción correcta es la concepción sintáctica; mientras que respecto a la aplicación de los enunciados jurídicos secundarios la concepción correcta es la concepción semántica.
\end{abstract}

Palabras clave: Aplicación del Derecho, motivación de las decisiones judiciales.
ABSTRACT. In the study of the judicial activity, the fundamental subject is not the motivation of the judicial decisions, as the current legal philosophy maintains, but the application of the legal sentences. And I think in addition that the standard conception of the motivation of the judicial decisions is erroneous precisely because of not to have considered this fact. On the other hand, I also consider mistaken the existing conceptions with respect to the application of the legal sentences (whether it is the theory of the judicial syllogism, or the logical conception). According to theses exposed in my book Las obligaciones básicas de los jueces (2005), with respect to the application of the primary legal sentences, the correct conception is the syntactic conception; whereas with respect to the application of the secondary legal sentences the correct conception is the semantic conception.

Key words: Application of the law, motivation of the judicial decisions.

En este trabajo desarrollo algunas ideas expuestas en mi libro Las obligaciones básicas de los jueces (Madrid, 2005), insistiendo en la conexión existente entre la aplicación de los enunciados jurídicos y el aspecto de la actividad judicial que constituye el centro de atención de los filósofos del Derecho desde hace décadas, la motivación de las decisiones judiciales.

Comenzaré ocupándome de este último tema. 


\section{La justificación de las decisiones judiciales, según N. MacCormick}

Examinemos el siguiente ejemplo:

[1] Quien compra una cosa está obligado a pagar su precio;

[2] Miguel compra el piso P;

[3] Miguel está obligado a pagar el precio del piso $\mathrm{P}$.

Supongamos que el enunciado [1] es un enunciado jurídico, un enunciado jurídico prescriptivo. Por otra parte, el enunciado [2] es un enunciado asertivo, que suponemos verdadero. Por último, supongamos también que, en un caso litigioso, se solicita a un juez que ordene a Miguel pagar el precio del piso P y, en respuesta a esa petición, el juez formula el enunciado prescriptivo [3], como decisión que resuelve el litigio.

La secuencia de enunciados [1] / [2] / [3] es un ejemplo de lo que se denomina «silogismo de la aplicación del Derecho», en el que: el enunciado [1] es la primera premisa, premisa mayor o premisa normativa; el enunciado [2] es la segunda premisa, premisa menor o premisa fáctica; y el enunciado [3], la decisión, es la conclusión deducida o deducible de ambas premisas.

Según N. MacCormick, en un caso sencillo, fácil, como el de este ejemplo, bastaría el razonamiento silogístico citado para que la decisión [3] quedara justificada. No obstante, en otros casos, calificables como difíciles, puede ocurrir según MacCormick que la justificación de una decisión tenga que afrontar problemas de cuatro tipos: de relevancia, interpretativos, de prueba y de calificación. Los dos primeros tipos de problemas, los de relevancia e interpretativos, tienen que ver con la premisa mayor del silogismo de la aplicación del Derecho (con el enunciado [1], en el ejemplo anterior); mientras que los de prueba y calificación tienen que ver con la premisa menor de dicho silogismo (con el enunciado [2], en el mismo ejemplo) ${ }^{1}$.

Estas ideas de MacCormick son bien conocidas y no es necesario exponerlas con más detalle. Lo que ahora deseo destacar del pensamiento de MacCormick son dos aspectos, que los comentaristas de MacCormick han pasado por alto y que a mí me parecen importantes.

El primero de ellos es que la obra de MacCormick no permite entender por qué la justificación de una decisión judicial consiste en realizar un razonamiento del tipo denominado «silogismo de la aplicación del Derecho» o en afrontar problemas interpretativos, de prueba, etc. ¿Por qué la justificación de una decisión judicial no consiste, por ejemplo, en mostrar o intentar mostrar que la decisión está bien redactada desde el punto de vista gramatical?

1 Véase Neil MacCormick: Legal Reasoning and Legal Theory, Oxford, Oxford University Press, 1995, capítulos III y IV. 
La segunda cuestión, que la obra de MacCormick tampoco permite comprender, es la de qué relación existe entre la justificación de una decisión judicial, o entre esos razonamientos o problemas que según MacCormick son propios de la justificación de las decisiones judiciales, y la aplicación de los enunciados jurídicos.

Una respuesta, aunque incompleta, a las cuestiones que acabo de plantear parece estar contenida en la concepción estándar del razonamiento justificatorio de las decisiones judiciales, de la que paso a ocuparme a continuación.

2. La concepción estándar del razonamiento justificatorio de las decisiones judiciales

\section{a) Exposición}

La que denomino «concepción estándar del razonamiento justificatorio de las decisiones judiciales» se caracteriza por sostener tres tesis que, aplicadas a la decisión [3] del ejemplo anterior, son las siguientes:

$1^{a}$ tesis) La justificación de la decisión [3] consiste en probar o intentar probar la tesis

[4] Existe un enunciado $E$ que es jurídico y un enunciado $F$ que es verdadero o está probado y tales que de la conjunción de $E$ y $F$ es deducible la decisión [3].

$2^{a}$ tesis) De la justificación o razonamiento justificatorio de la decisión [3] forma parte un silogismo de la aplicación del Derecho. Éste es condición necesaria para la justificación de la decisión [3].

$3^{a}$ tesis) La conclusión final del razonamiento justificatorio de la decisión [3] es la propia decisión [3].

La relación que existe entre estas tres tesis parece ser la siguiente:

La prueba de la tesis [4] requiere probar la existencia de dos enunciados $E$ y $F$, tales que, por un lado,

[5] El enunciado $E$ es un enunciado jurídico y el enunciado $F$ es verdadero o está probado;

$\mathrm{y}$, por otro lado,

[6] De $E$ y $F$ es deducible la decisión [3].

La prueba de estas tesis [5] y [6] y, en definitiva, la prueba de la tesis [4] exige, por tanto, probar la existencia de dos enunciados determinados, que pueden 
ser los enunciados [1] («Quien compra una cosa está obligado a pagar su precio») y [2] («Miguel compra el piso $\mathrm{P} »)$, que tienen las propiedades a que aluden las tesis [5] y [6]. Hay que justificar, por ejemplo, las dos tesis siguientes:

[7] El enunciado [1] es un enunciado jurídico y el enunciado [2] es verdadero o está probado

$\mathrm{y}$

[8] De [1] y [2] es deducible la decisión [3].

Conforme a la terminología introducida por J. Wróblewski y consolidada en la filosofía del Derecho, la prueba de la tesis [7] sería denominada «problema de la justificación externa de la decisión [3]»; mientras que la prueba de la tesis [8] sería denominada «problema de la justificación interna de la decisión [3]»². Aunque, a mi juicio, sería preferible denominar la prueba de la tesis [7] «problema externo de la justificación de la decisión [3]»; y la prueba de la tesis [8], «problema interno de la justificación de la decisión [3]».

Según la concepción estándar del razonamiento justificatorio, el razonamiento para probar la tesis [8] es el silogismo de la aplicación del Derecho; concretamente, el razonamiento, cuyas premisas son los enunciados [1] y [2], y cuya conclusión es la decisión [3]. Este razonamiento es necesario y suficiente para probar la citada tesis [8], o sea, para la justificación interna de la decisión [3] ${ }^{3}$.

Por otro lado, la prueba de la tesis [8], la justificación interna de la decisión [3], es condición necesaria para probar la tesis [4]. Y, según la primera de las tesis de la concepción estándar, probar la tesis [4] es condición necesaria (y también suficiente) para justificar la decisión [3]. De ahí la conclusión de que el silogismo de la aplicación del Derecho, considerado necesario y suficiente para la justificación interna de la decisión [3], sea considerado (meramente) necesario para la justificación de la decisión [3]. Esta conclusión es la que está recogida en la segunda tesis que caracteriza a la concepción estándar.

2 Véase, por ejemplo, Jerzy Wróblewski: «Legal Decision and Its Justification», en Logique et analyse, vol. 53/54 (1971), pp. 409-419, p. 412; Jerzy Wróblewski: «Motivation de la décision judiciaire», en Ch. Perelman et P. Foriers (Études publiées par): La motivation des décisions de justice, Bruxelles, Émile Bruylant, 1978, pp. 111-135, pp. 119-120.

3 Esto es lo que se deduce claramente del siguiente texto de Aulis Aarnio: Derecho, Racionalidad y Comunicación Social. Ensayos de Filosofía del Derecho, traducción de Pablo Larrañaga, México, Distribuciones Fontamara, 2004, p. 55: «El modelo clásico de la justificación interna es el silogismo aristotélico (modus ponens). La primera premisa es la "base normativa" de la decisión (premisa normativa). La forma lógica de la deducción garantiza que la conclusión, la norma de la decisión, se siga de las premisas». Véase también Robert Alexy: Teoría de la argumentación jurídica. La teoría del discurso racional como teoría de la argumentación jurídica, traducción de Manuel Atienza e Isabel Espejo, Madrid, Centro de Estudios Constitucionales, 1997, pp. 213-214. 
La concepción estándar sostiene así que el silogismo de la aplicación del Derecho está incluido en el razonamiento justificatorio de la decisión [3]. Por otra parte, la conclusión del silogismo de la aplicación del Derecho es, en el ejemplo actual, dicha decisión [3]. De todo ello la concepción estándar infiere que la conclusión final del razonamiento justificatorio de la decisión [3] también es la propia decisión [3], como sostiene la tercera de las tesis que caracterizan a dicha concepción ${ }^{4}$.

\section{B) Comentarios}

Voy a hacer a continuación dos comentarios a la concepción que acabo de exponer, a la concepción estándar del razonamiento justificatorio.

\section{Comentario $1^{\circ}$ )}

El primer comentario es el siguiente.

Si comparamos la concepción estándar del razonamiento justificatorio, tal como ha sido expuesta, con las tesis de MacCormick antes examinadas, podríamos decir que la concepción estándar del razonamiento justificatorio no distingue entre casos fáciles y difíciles; y que, según esta concepción, en todos los casos, para que una decisión judicial quede completamente justificada, es necesario realizar un razonamiento deductivo, un silogismo de la aplicación del Derecho, a fin de justificar internamente la decisión, y también es necesario afrontar los cuatro tipos de problemas destacados por MacCormick (de relevancia, interpretativos, de prueba y de calificación), a fin, como se suele decir, de justificar las premisas de dicho silogismo, a fin de justificar externamente la decisión.

Yo creo que, aunque aceptemos la primera de las tesis de la concepción estándar del razonamiento justificatorio, la segunda y la tercera tesis de esta concepción son erróneas, por razones que he expuesto detenidamente en el libro mío que antes he citado, y que aquí voy a resumir.

\footnotetext{
4 Así, la STS de 20 de octubre de 1995 dice, en su fundamento de Derecho primero, que «la motivación es una exigencia formal de las sentencias, en cuanto deben expresar las razones de hecho y de derecho que las fundamentan, es decir, el proceso lógico-jurídico que conduce a la decisión o fallo». Eugenio Bulygin: «Sentencia judicial y creación del Derecho», en Carlos E. Alchourrón y E. Bulygin: Análisis lógico y Derecho, Madrid, Centro de Estudios Constitucionales, 1991, pp. 355-369, p. 356: «Justificar o fundar una decisión consiste en construir una inferencia o razonamiento lógicamente válido, entre cuyas premisas figura una norma general y cuya conclusión es la decisión». Stefano Evangelista: «Motivazione della sentenza civile», en Enciclopedia del diritto, vol. XXVII, pp. 154-180, p. 160: «(la ley), al describir la forma en que la sentencia se presenta exteriormente, impone que la motivación preceda al dispositivo, haciendo aparecer este último como la conclusión silogística de las premisas establecidas con la primera». Francesco M. Iacoviello: La motivazione della sentenza penale e il suo controllo in cassazione, Milano, Giuffrè, 1997, p. 294: «Partiendo de la idea de la sentencia como discurso unitario compuesto de premisas (la motivación) y conclusión (el dispositivo) ...». Fernando Zubiri de Salinas: «La motivación de las sentencias», en Cuadernos del Derecho judicial. La sentencia penal, Madrid, Consejo General del Poder Judicial, 1992, pp. 273-284, p. 276: «la motivación consiste en un razonamiento que conduce al fallo». Véase también, en el mismo sentido, Paolo Comanducci: «La motivazione in fatto», en Giulio Ubertis (a cura di): La conoscenza del fatto nel processo penale, Milano, Giuffrè, 1992, pp. 215-244, p. 217.
} 
En cuanto a la tercera tesis, la tesis de que la conclusión final del razonamiento justificatorio de la decisión [3] es la propia decisión [3], esta tesis choca, a mi juicio, con la primera y más importante tesis de la concepción estándar. Choca con la tesis que afirma que la justificación de la decisión [3] («Miguel está obligado a pagar el precio del piso $\mathrm{P} \gg$ ) consiste en probar o intentar probar la tesis

[4] Existe un enunciado $E$ que es jurídico y un enunciado $F$ que es verdadero o está probado, y tales que de la conjunción de $E$ y $F$ es deducible la decisión [3].

Pues si justificar la decisión [3] consiste en (intentar) probar la tesis [4], es esta tesis [4], y no la propia decisión [3], la que debería ser la conclusión final del razonamiento justificatorio de dicha decisión. Y ello aunque aceptemos la segunda tesis de la concepción estándar, o sea, la tesis de que, para justificar la decisión [3], es necesario realizar un silogismo de la aplicación del Derecho, como la secuencia de enunciados [1] / [2] / [3], cuya conclusión es dicha decisión [3].

Paso ahora a comentar esta segunda tesis.

La segunda tesis de la concepción estándar es consecuencia de dos tesis sostenidas por esta concepción:

Una de ellas es la que denomino $\ll 1^{a}$ tesis» de dicha concepción y que acabo de recordar.

La otra es la tesis que afirma que, para probar que la decisión [3] es deducible de dos enunciados $E$ y $F$, es necesario y suficiente realizar un razonamiento, cuyas premisas sean esos enunciados $E$ y $F$, y cuya conclusión sea dicha decisión [3]. Conforme a esta tesis, y dicho más concretamente, el silogismo de la aplicación del Derecho citado al comienzo

[1] Quien compra una cosa está obligado a pagar su precio

[2] Miguel compra el piso $\mathrm{P}$

[3] Miguel está obligado a pagar el precio del piso $\mathrm{P}$,

cuyas premisas son los enunciados [1] y [2], y cuya conclusión es la decisión [3], es necesario y suficiente para probar que [3] es deducible de [1] y [2]. O sea, necesario y suficiente para la justificación interna de la decisión [3]; necesario y suficiente probar la tesis

[8] De [1] y [2] es deducible la decisión [3],

o la tesis

[9] Del enunciado prescriptivo [1] y del enunciado asertivo [2] es deducible la decisión prescriptiva [3]. 
Sin embargo, a mi juicio, es un error creer que la secuencia de enunciados [1] / [2] / [3] es necesaria o suficiente para probar la tesis [8] o la tesis [9].

Pues fijémonos en la siguiente secuencia de enunciados:

[10] Todos los hombres son mortales;

[11] Aquiles es hombre;

[12] Aquiles es mortal.

Esta secuencia de enunciados no es necesaria, ni suficiente para probar la tesis

[13] De [10] y [11] es deducible [12].

Hay que subrayar, en particular, que esta tesis [13] no es una consecuencia de la secuencia de enunciados [10] / [11] / [12]. La tesis [13], dicho sea incidentalmente, es una consecuencia de las nociones de deducibilidad (o consecuencia lógica / consecuencia semántica) y verdad. Y el que la tesis [13] sea verdadera es lo que permite contemplar la secuencia de enunciados [10] / [11] / [12] como un razonamiento deductivo.

Por las mismas razones, tampoco la secuencia de enunciados [1] / [2] / [3] es necesaria o suficiente para probar la tesis [8] o la tesis [9]. Es necesario destacar, frente a una creencia muy extendida, que la tesis [8] o la tesis [9], en cuya justificación consiste la justificación interna de la decisión [3], no es una consecuencia del silogismo de la aplicación del Derecho, o sea, de la secuencia de enunciados [1] / [2] / [3]. Pero, a diferencia de la tesis [13], y dicho sea de nuevo incidentalmente, la tesis [8] o la [9] no es una consecuencia de las nociones de deducibilidad y verdad, ni tampoco es consecuencia de ninguna otra cosa. La tesis [8] o la [9] es una afirmación gratuita. No existe ninguna razón para pensar que es una tesis verdadera. Y menos razón hay aún para pensar que es una tesis verdadera y que, por ello, el silogismo de la aplicación del Derecho, la secuencia de enunciados [1] / [2] / [3], es una inferencia deductiva.

Puesto que, según cuanto acabo de decir, el silogismo de la aplicación del Derecho no es necesario ni suficiente para justificar internamente una decisión judicial, dicho silogismo tampoco es necesario, a mi juicio, para la justificación de una decisión. Y ello aunque aceptemos la primera tesis de la concepción estándar del razonamiento justificatorio. Esta concepción había subrayado que el lugar del silogismo de la aplicación del Derecho no se haya en el proceso decisorio del juez, en el contexto de descubrimiento, sino en el proceso de justificación, en el contexto de justificación. En mi opinión, en cambio, el silogismo de la aplicación del Derecho es irrelevante tanto en el proceso decisorio, como en el proceso justificatorio. Las prescripciones o normas, dicho sea respondiendo a una observación que M. Atienza me hace en su último libro ${ }^{5}$, no son parte necesaria del discurso argumentativo

5 Manuel Atienza: El Derecho como argumentación, Barcelona, Ariel, 2006, p. 131. 
del juez, ni como premisas ni como conclusiones. Sólo son el objeto al que dichas premisas y conclusiones se refieren.

Las tesis de la concepción estándar del razonamiento justificatorio que acabo de criticar (es decir, la creencia de que el silogismo de la aplicación del Derecho es necesario para la justificación de una decisión judicial, así como la tesis, independiente de la anterior, de que la conclusión final del razonamiento justificatorio de una decisión es la propia decisión, decisión que en la mayoría de los casos es un enunciado prescriptivo) son las que más han impulsado el tema de la argumentación jurídica o del razonamiento jurídico, como una forma de razonamiento que es especial en algún sentido no precisado.

\section{Comentario $2^{\circ}$ )}

Mi segundo comentario a la concepción estándar del razonamiento justificatorio se va a referir a las cuestiones que antes he planteado con relación al pensamiento de MacCormick, y tienen que ver con la primera de las tesis de dicha concepción.

Una de las cuestiones que he planteado, al hablar de MacCormick, es la de por qué el silogismo de la aplicación del Derecho y los problemas interpretativos, de prueba, etc., tienen que ver con la justificación de una decisión judicial. La concepción estándar respondería a esta cuestión de la manera siguiente: el silogismo de la aplicación del Derecho es necesario (y suficiente) para justificar internamente la decisión judicial, mientras que afrontar los problemas interpretativos, de prueba, etc., es necesario para justificar externamente la decisión; y, para que una decisión judicial esté plenamente justificada, es necesario que la decisión esté justificada, tanto interna como externamente.

Esta respuesta es una consecuencia de la que vengo denominando «primera tesis de la concepción estándar del razonamiento justificatorio». Es una consecuencia de la tesis que afirma que justificar la decisión [3] («Miguel está obligado a pagar el precio del piso $\mathrm{P} »)$ consiste en probar o intentar probar la tesis

[4] Existe un enunciado $E$ que es jurídico y un enunciado $F$ que es verdadero o está probado, y tales que de la conjunción de $E$ y $F$ es deducible la decisión [3].

Sin embargo, la concepción estándar no proporciona ninguna explicación de esta tesis, tesis fundamental, suya. ¿Por qué, para justificar la decisión [3], hay que probar o intentar probar que un enunciado (como el enunciado [1], «Quien compra una cosa está obligado a pagar su precio») es jurídico? ¿Por qué, para justificar la decisión [3], hay que probar o intentar probar que un enunciado (como el enunciado [2], «Miguel compra el piso $\mathrm{P}$ ») es verdadero o está probado?; ¿por qué, dicho de otro modo, el razonamiento probatorio es necesario para la justificación de una decisión judicial? ¿Por qué, finalmente, para justificar la decisión [3], hay que probar o intentar probar que dicha decisión es deducible de dos enunciados, como los enunciados [1] y [2]? 
La concepción estándar del razonamiento justificatorio no responde a ninguna de estas preguntas. Por ello, tampoco puede responder a esta otra pregunta, que antes he formulado: ¿por qué la justificación de una decisión judicial no consiste en probar o intentar probar que la decisión judicial está bien redactada desde el punto de vista gramatical?

A mi juicio, las respuestas a las preguntas anteriores, en particular la explicación de la primera de las tesis de la concepción estándar, se halla en las dos tesis siguientes:

[14] Justificar la decisión [3] consiste en (intentar) probar que [3] aplica un enunciado jurídico;

[15] La decisión [3] aplica un enunciado jurídico si, y sólo si, existe un enunciado $E$ que es jurídico y un enunciado $F$ que es verdadero o está probado, y tales que de la conjunción de $E$ y $F$ es deducible [3].

De estas dos tesis, [14] y [15], se deduce que

[16] Justificar la decisión [3] consiste en (intentar) probar que existe un enunciado $E$ que es jurídico y un enunciado $F$ que es verdadero o está probado, y tales que de la conjunción de $E$ y $F$ es deducible [3].

Y esta tesis [16] es precisamente la primera tesis de la concepción estándar. Pues recordemos que lo que esta primera tesis dice es que justificar la decisión [3] consiste en (intentar) probar que

[4] Existe un enunciado $E$ que es jurídico y un enunciado $F$ que es verdadero o está probado, y tales que de la conjunción de $E$ y $F$ es deducible la decisión [3].

Observamos así que las tesis [14] y [15], por un lado, justifican la primera tesis de la concepción estándar y permiten también responder a las preguntas, antes formuladas, de por qué, para justificar la decisión [3], es necesario (intentar) probar que un determinado enunciado (como el enunciado [1]) es jurídico y también es necesario el razonamiento probatorio.

Por otro lado, esas mismas dos tesis [14] y [15], concretamente, la tesis [14], también contienen una respuesta a otro problema planteado al comentar las tesis de MACCoRmick: el de cuál es la relación existente entre la aplicación de los enunciados jurídicos y la motivación de las decisiones judiciales. Pues justificar una decisión judicial consiste, según la tesis [14], en (intentar) probar que la decisión aplica un enunciado jurídico.

Mas, pasando de la exposición a la crítica, ¿son correctas esas tesis [14] y [15], que permiten explicar la primera y fundamental tesis de la concepción estándar? 
La tesis [15] expresa una concepción de la aplicación de los enunciados jurídicos, la concepción lógica, que a mi juicio es falsa. Una falsedad que resulta evidente cuando el enunciado jurídico de cuya aplicación se trata es un enunciado secundario (esto es, un enunciado que dice cómo la Administración Pública o los jueces deben decidir o decidirán un determinado asunto). Aunque también es posible probar que dicha concepción es falsa cuando el enunciado jurídico de cuya aplicación se trata es un enunciado primario (esto es, o bien un enunciado prescriptivo, cuyos destinatarios no son ni la Administración Pública ni los jueces, o bien un enunciado cualificatorio). También es falsa, dicho sea incidentalmente, la que, en el libro mío citado al comienzo de este trabajo, denomino «tesis lógica de los tribunales», tesis que afirma que la decisión que aplica un enunciado jurídico es deducible de los antecedentes de hecho y de los fundamentos de Derecho de la Sentencia que contiene la decisión; pues ninguna, absolutamente ninguna, decisión judicial, contenida en una Sentencia "se basa en", en el sentido de "es deducible de", los antecedentes de hecho y los fundamentos de Derecho de la Sentencia que contiene la decisión. Según las ideas expuestas en ese libro mío, las concepciones correctas de la aplicación de los enunciados jurídicos son las que denomino «concepción sintáctica», tratándose de la aplicación de enunciados jurídicos primarios, y «concepción semántica», si se trata de la aplicación de enunciados jurídicos secundarios.

En cuanto a la tesis [14], también su verdad es cuestionable. ¿Qué razón hay para pensar qué justificar una decisión consiste en (intentar) probar que la decisión aplica un enunciado jurídico?¿Por qué justificar una decisión judicial no consiste, como sostienen algunos autores, en intentar persuadir a un determinado auditorio, o en probar o intentar probar que la decisión es justa o moralmente correcta, o en probar o intentar probar que la decisión tiene consecuencias sociales deseables, o es universalizable, o es la conclusión de un procedimiento discursivo racional? O incluso, dicho sea repitiendo una vez más la misma pregunta, ¿por qué justificar una decisión judicial no consiste en probar o intentar probar que la decisión está bien redactada desde el punto de vista gramatical?

Son posibles, pues, y de hecho existen diversas concepciones de la justificación de las decisiones judiciales, diferentes de la concepción contenida en la tesis [14]. Y el problema que se plantea es el de cómo determinar cuál de esas concepciones es la correcta.

A primera vista, la concepción encerrada en la tesis [14] presenta dos características ventajosas sobre las demás concepciones citadas. Por un lado, dicha concepción o dicha tesis [14], unida a la tesis [15], es la única que explica las tesis de la concepción estándar de la justificación de las decisiones judiciales, las tesis de esta concepción acerca de la justificación interna y la justificación externa de las decisiones. Por otra parte, la concepción contenida en la tesis [14] es la única que relaciona la justificación de las decisiones judiciales y la aplicación de los enunciados jurídicos. 
Mas, ¿por qué estas características, que indudablemente presenta exclusivamente la tesis [14], son ventajas?

El que la tesis [14] permita explicar un tópico, como es en definitiva la concepción estándar del razonamiento justificatorio, en particular, la primera tesis de esta concepción, no es un argumento suficiente a favor de la tesis [14], a no ser que ese tópico sea verdadero. Mas esto, o sea, la verdad de dicho tópico, es precisamente lo que se está cuestionando. Dicho más claramente, es cierto que, como hemos observado, la tesis [14] permite explicar la primera de la tesis de la concepción estándar, o sea, la tesis

[16] Justificar la decisión [3] consiste en (intentar) probar que existe un enunciado $E$ que es jurídico y un enunciado $F$ que es verdadero o está probado, y tales que de la conjunción de $E$ y $F$ es deducible [3].

Mas, para que esa potencia explicativa de la tesis [14] fuera un argumento a su favor, sería necesario que fuera verdadero el explicandum, o sea, la tesis [16]. Pero esto, es decir, la verdad de la tesis [16], es precisamente lo que está en tela de juicio.

Por otra parte, también habría que explicar por qué es una ventaja de la tesis [14] el que esta tesis relacione la justificación de las decisiones judiciales y la aplicación de los enunciados jurídicos.

A mi juicio, si planteamos la cuestión que nos ocupa en los términos en que lo acabamos de hacer, es decir, si nos preguntamos simplemente qué es justificar una decisión judicial, creo que es muy difícil, si no imposible, hallar argumentos determinantes para preferir una determinada concepción de la justificación de las decisiones judiciales a las demás concepciones.

\section{Un problema interpretativo}

Sin embargo, considero que plantear dicha cuestión en los términos citados es un planteamiento incorrecto.

El término «justificar» es un término usado por las doctrinas o teorías, en lugar de «motivar», que es el término que suele usar el legislador en los enunciados jurídicos que ordenan a los jueces motivar sus decisiones (sus Sentencias o Resoluciones, dice impropiamente el legislador). Son estos enunciados jurídicos y el término «motivar» contenido en ellos los que durante las últimas décadas han propiciado el interés por el estudio de la motivación o justificación de las decisiones judiciales. Por ello, la pregunta que estas investigaciones deberían plantear es un problema de interpretación del Derecho: el problema de determinar qué significa el término «motivar» en los enunciados jurídicos que ordenan a los jueces motivar sus decisiones. 
Planteada la cuestión de esta manera, creo que sería falso responder a esa pregunta diciendo que, en los contextos citados, el término «motivar (una decisión)» significa «intentar persuadir a los ciudadanos (o a tal público o auditorio) a fin de que acepten una decisión»; o «intentar mostrar que la decisión es justa (es razonable / tiene consecuencias deseables / es universalizable / es moralmente correcta)», o «intentar mostrar que la decisión es la conclusión de una argumentación correcta según determinadas reglas (no jurídicas)».

«Motivar una decisión» significa «indicar el motivo por el que ha sido dictada una decisión». Y, siendo obligación de los jueces dictar decisiones que sean conformes al Derecho, hay que presumir que el motivo por el que ha sido dictada una determinada decisión $X$, en vez de otra decisión distinta, consiste en que el juez que ha dictado $X$ considera que dicha decisión es conforme con el Derecho.

Este motivo, pues, hay que darlo por descontado. Mas por ello, precisamente, el cumplimiento de la obligación jurídica de motivar una decisión judicial $X$ no puede quedar reducido a realizar la manifestación, que sería igual para todas las decisiones judiciales, de que $X$ ha sido dictada por ser, a juicio del juez que la ha dictado, conforme al Derecho. El juez que ha de motivar una decisión $X$ ha de ir más allá de ese motivo. Ha de indicar cuál es el motivo de ese motivo. Cuando el Derecho establece que el juez que dicta una decisión debe motivarla, lo que le está exigiendo es que indique el motivo por el que él considera que dicha decisión es conforme al Derecho. En otras palabras, la obligación de motivar exige al juez que dicta una decisión $X$ que intente mostrar que $X$ es conforme al Derecho.

La motivación de una decisión judicial consiste, pues, en mi opinión, en un intento de mostrar o probar que la decisión que ha sido dictada es conforme al Derecho. Y si entendemos que la corrección de una decisión judicial consiste en su conformidad al Derecho, podemos decir también que la motivación de una decisión judicial consiste en un intento de mostrar o probar que la decisión es correcta.

Por otro lado, en toda decisión judicial es posible distinguir dos aspectos: el aspecto material o de contenido, y el aspecto procedimental o procesal. Por ello, en la motivación de una decisión judicial también podemos distinguir dos aspectos: un intento de mostrar que la decisión es materialmente correcta (materialmente conforme al Derecho) y un intento de mostrar que la decisión es procesalmente correcta (procesalmente conforme al Derecho).

A efectos de enjuiciar si una decisión judicial es procesalmente correcta, es indiferente de qué tipo de decisión se trate. Es indiferente, dicho con más precisión, si se trata de una decisión estimatoria o desestimatoria.

Pero considero que la distinción entre estas dos categorías de decisiones judiciales (distinción, dicho sea incidentalmente, que los estudios filosófico-jurídicos sobre la actividad judicial pasan por alto) sí es relevante para enjuiciar la corrección material de una decisión judicial. Y ello por las razones siguientes: 
Una decisión es materialmente correcta, materialmente conforme con el Derecho, si y sólo si la decisión tiene el contenido que según el Derecho debe tener.

Por otra parte, una decisión judicial tiene el contenido que según el Derecho debe tener si, y sólo si, la decisión dice el Derecho (pues lo que el Derecho, concretamente, el art. 117 de la Constitución Española de 1978, exige a las decisiones judiciales sólo es que éstas digan el Derecho. Así interpreto este precepto constitucional, que establece que los jueces no tienen más función que la función jurisdiccional, al juzgar y ejecutar lo juzgado).

De estas observaciones se deduce que una decisión es materialmente correcta si, y sólo si, la decisión dice el Derecho, el Derecho material o el Derecho procesal. (Conviene subrayar que el que la decisión diga, o no diga, el Derecho procesal condiciona la corrección material de la decisión, no su corrección procesal).

Un corolario de esta conclusión, de la conclusión de que una decisión materialmente correcta es la que dice el Derecho, es que la justificación o motivación de la corrección material de una decisión (su conformidad material al Derecho) consiste en intentar mostrar o probar que la decisión dice el Derecho.

Ahora bien, en mi opinión, las decisiones estimatorias y las desestimatorias no siempre dicen el Derecho de la misma manera. Y ésta es la razón por la que la distinción entre estas dos categorías de decisiones judiciales es relevante a la hora de analizar la motivación de la corrección material de las decisiones judiciales.

A mi juicio, tratándose de decisiones estimatorias, decir el Derecho equivale a aplicar el Derecho.

En cambio, una decisión desestimatoria (formulada mediante locuciones como «desestimo la pretensión ...», «no hay lugar a la demanda ...», «se absuelve al acusado», etc.) puede decir el Derecho de dos maneras: aplicando el Derecho procesal, o sea, aplicando un enunciado jurídico procesal, o bien por el mero hecho de que ninguna decisión estimatoria de la petición que el juez ha recibido diría o aplicaría el Derecho material.

Todas estas observaciones implican la conclusión siguiente: motivar una decisión judicial consiste, por un lado, en intentar probar que la decisión es procesalmente correcta (procesalmente conforme al Derecho); y también, por otro lado, en intentar probar que la decisión aplica el Derecho, si es que se trata de una decisión estimatoria; o bien, en el caso de que la decisión sea desestimatoria, en intentar probar o que la decisión aplica el Derecho (el Derecho procesal) o que ninguna decisión estimatoria aplicaría el Derecho (el Derecho material).

Esta conclusión tiene un corolario que afecta a la tesis

[14] Justificar la decisión [3] consiste en (intentar) probar que [3] aplica un enunciado jurídico,

que subyace a la concepción estándar de la justificación de las decisiones judiciales. 
Pues, conforme a la conclusión que acaba de ser alcanzada, esta tesis [14] es correcta, siempre que se hagan las tres matizaciones siguientes:

- La primera es que habría que aceptar la tesis, más polémica de lo que a primera vista pudiera parecer, de que aplicar el Derecho es lo mismo que aplicar un enunciado jurídico;

- La segunda es que se precise que la decisión [3], a la que la tesis [14] alude, es una decisión estimatoria; y

- La tercera es que la justificación a la que la tesis [14] se refiere es la justificación de la corrección material de la decisión [3].

Hechas estas matizaciones, la tesis [14] resulta correcta. Dicho de otro modo, habría que sustituir la tesis [14] por esta otra más exacta:

[17] Justificar la corrección material de la decisión [3], que es una decisión estimatoria, consiste en (intentar) probar que [3] aplica un enunciado jurídico.

Por otra parte, esta tesis [17] revela cuál es la conexión entre la aplicación de los enunciados jurídicos y la motivación de las decisiones judiciales o, más precisamente, cuál es la relación entre la aplicación de los enunciados jurídicos y la motivación o justificación de la corrección material de una decisión estimatoria.

Pero la noción de aplicación de un enunciado jurídico también es relevante en el caso de que la decisión cuya corrección material haya que justificar sea una decisión desestimatoria. Pues, como antes he dicho, justificar la corrección material de una decisión desestimatoria consiste en intentar probar o bien que la decisión aplica el Derecho (el Derecho procesal) o bien que ninguna decisión estimatoria aplicaría el Derecho (el Derecho material).

Todo ello revela que la noción de aplicar el Derecho, de aplicar los enunciados jurídicos, es una noción más primaria o elemental que la de motivar las decisiones judiciales, dado que esta segunda se define en última instancia mediante la primera.

Sin embargo, paradójicamente, la aplicación de los enunciados jurídicos es un tema casi totalmente ignorado. Los estudios de las últimas décadas, centrados en la motivación o justificación de las decisiones judiciales, han saltado por encima del tema de la aplicación de los enunciados jurídicos; lo que me parece que es poner el carro delante de los bueyes. Y la consecuencia es que, a pesar de la abundantísima literatura existente sobre la actividad judicial, con relación a la aplicación de los enunciados jurídicos, el análisis realizado por $\mathrm{C}$. Beccaria hace más de dos siglos sea lo más riguroso de que aún hoy disponemos. La aplicación de los enunciados jurídicos sigue siendo contemplada a la luz de la teoría del silogismo de la aplicación del Derecho y sigue siendo expuesta en términos parecidos a los usados por Beccaria: se habla de la aplicación de los 
enunciados jurídicos, como un razonamiento silogístico, pero no se define qué es una decisión que aplica un enunciado jurídico, ni se plantea la cuestión de cómo distinguir las decisiones judiciales que aplican un enunciado jurídico determinado de las decisiones que no lo aplican. Éste es, en mi opinión, el primer problema que hay que afrontar antes de ocuparse, mucho después, de la motivación de las decisiones judiciales. El análisis de la motivación de las decisiones judiciales exige haber analizado previamente un gran número de temas, con autonomía propia, y el primero de ellos es, como digo, el de la aplicación de los enunciados jurídicos. 